\title{
IL RiCHiAMo DELLE LETTERE: \\ Lingua, Cultura e Identità nelle \\ CORRISPONDENZE EPISTOLARI DEI MigRanti Italiani in Brasile
}

Federico Croci

ABSTRACT Fra il secolo XIXeXX, un milione e mezzo di italiani sono emigrati in Brasile. Si tratta di un fenomeno che ha costretto i suoi protagonisti a vivere lunghe, dolorose e, spesso, definitive separazioni dai familiari e dalle loro comunità. Un'esperienza che è cominciata con la traversata oceanica, una specie di rito di passaggio nel quale si concentra simbolicamente la condizione dell'emigrante, caratterizzata dal senso di sradicamento. In un modo più preciso, i segni tangibili di questo processo di frantumazione dell'identità e dei tentativi di ricomposizione, realizzati con difficoltà ma anche con grande ostinazione, sono le lettere che consentono di ristabilire un punto di continuità col passato e con la comunità di origine. Le navi tagliavano i mari con merci e uomini, seguite da una scia di parole e di scrittura che oggi rappresenta una testimonianza preziosa e, per certi aspetti, insostituibile per la ricostruzione di momenti della storia della lingua italiana nel suo contatto con la lingua portoghese. In questo articolo si analizza una tipologia particolare di queste lettere: le lettere di chiamata. Interferenze linguistiche e aspetti sociolinguistici della lingua scritta in questi documenti sono un prisma valido per analizzare interscambi decorrenti dal contatto fra le due culture.

PAROLE CHIAVE immigrazione italiana; sociolinguistica; scrittura popolare; lettere di chiamata. 
lilit (11) Entre o século XIXeXX, um milhão e meio de italianos emigraram ao Brasil. Tratase de um fenômeno que obrigou seus protagonistas a viver longas, dolorosas e, freqüentemente, definitivas separações de familiares e das próprias comunidades. Uma experiência que começou com a travessia oceânica, uma espécie de ritual de passagemno qual se concentra simbolicamente a condição do migrante, caracterizada pelo sentido de desarraigamento. Mais precisamente, os sinais tangíveis deste processo de fragmentação da identidade e das tentativas de recomposição, cansativamente mas obstinadamente realizadas, são as cartas que permitem restabelecer um ponto de continuidade com o passado e com a própria comunidade de origem. Os navios singravam os mares com mercadorias e homens, acompanhados por uma esteira de palavras e de escritos que, hoje, constituem um testemunho precioso e, por alguns aspectos, insubstituivel para tentar reconstruir momentos da história da lingua italiana no seu contato com a língua portuguesa. Nesse artigo, analisa-se uma tipologia particular dessas cartas: as cartas de chamada. Interferências lingüísticas e aspectos sociolingüísticos da língua escrita nesses documentos constituem um prisma valioso para analisar intercâmbios devidos ao contato entre as duas culturas.

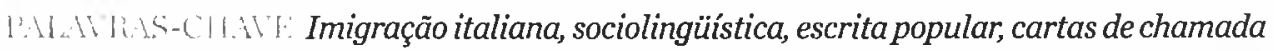

IFSTIACT Between the 19th and 20th century, one and a half million Italians emigrated to Brazil. The move forced most to lead long, painful lives and often permanent separation from family members and their own communities. The experience began with an ocean crossing, a sort of rite of passage in which the position of migrants is symbolically represented by the concept of uprooting. More accurately, the tangible signs of this process of identity fragmentation followed by tiresome but stubborn attempts at rebuilding it are seen in the letters. They restore a connection with the past and their own community of origin. The ships sailed the seas with goods and crews, trailing a wake of words and writings that today make up an invaluable and somewhat irreplaceable source of information if one is to try and rebuild the historic encounter between the Italian and the Portuguese languages. This paper looks into a unique typology among these letters: the cartas de chamada (invitation letters). The language interferences and socio-linguistic aspects of the written language found in these documents provide a precious perspective into the identity and cultural exchanges that resulted from this contact. $\mathrm{KIIIV}$ Italian immigration, socio-linguistics, popular writings, invitation letters 
Jaco Spina - S'io fossi re, - disse, e sputò - s'io fossi re, nemmeno una lettera, ma no! che dico una lettera!, nemmeno un semplice saluto farei più arrivare a Fàrnia da laggiù!

La Gialluzza-Bravozi' Jaco Spina; e come farebbero qua le povere mamme, le spose, senza notizie e senz'ajuto? Jaco Spina - Sí! Ne mandano assai! - e sputò di nuovo - Le madri, a far le serve, e le spose vanno a male! Su certe case le corna vedo crescere fino ai sette cieli! Vorrei sapere perché il male che trovano laggiù non lo dicono nelle loro lettere. Solo il bene dicono. Eogni lettera che arriva è qua per questi ragazzacci ignoranticome la chioccia: - pio, pio, pio-se lichiama e porta via tutti quanti!

Luigi Pirandello, L'altro figlio, 1923

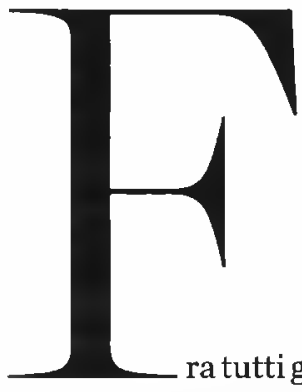
la lettera è quello che racchiude in sé un potenziale evocativo e di fascinazione di portata eccezionale, per il semplice fatto di essere un documento privato e personale che, almeno virtualmente, ci trasporta all'improvviso nel bel mezzo dell'evento emigrazione permettendoci di osservarne alcuni aspetti o momenti, anche particolarmente intimi, dall'interno, attraverso il punto di vista dei protagonisti. Il potere di suggestione della corrispondenza privata si deve proprio a questa contaminazione fra vita intima e quotidiana - nella quale ognuno di noi può riconoscersi - e l'impronta diretta della Storia, il passaggio del tempo nella soggettività, senza rielaborazioni e adattamenti della memoria. Si tratta indubbiamente di una visione parziale, sottoposta ai molteplici condizionamenti e filtri ai quali ogni singolo autore di questi documenti è vincolato, ma che ci consente di addentrarci nell'esplorazione delle tracce che le trasformazioni sociali, culturali e identitarie indotte dal fenomeno migratorio hanno lasciato.

Ogni storia d'emigrazione inizia con una partenza, con un distacco, è la lontananza che produce bisogno di comunicazione e la comunicazione a distanza, in quell'epoca - ossia quella della Grande Emigrazione, che grosso modo, per convenzione, viene considerata quella compresa nel periodo che va dal 1876 al 1914 - e almeno fino all'invenzione e diffusione del telefono, poteva solo essere 
scritta. Quando i bastimenti carichi di emigranti stavano per partire vi era l'usanza che coloro i quali s'imbarcavano tenessero in mano un filo di lana, l'altro capo del quale rimaneva in mano ai parenti che restavano sul molo e lo stringevano fra le dita fino all'ultimo momento.

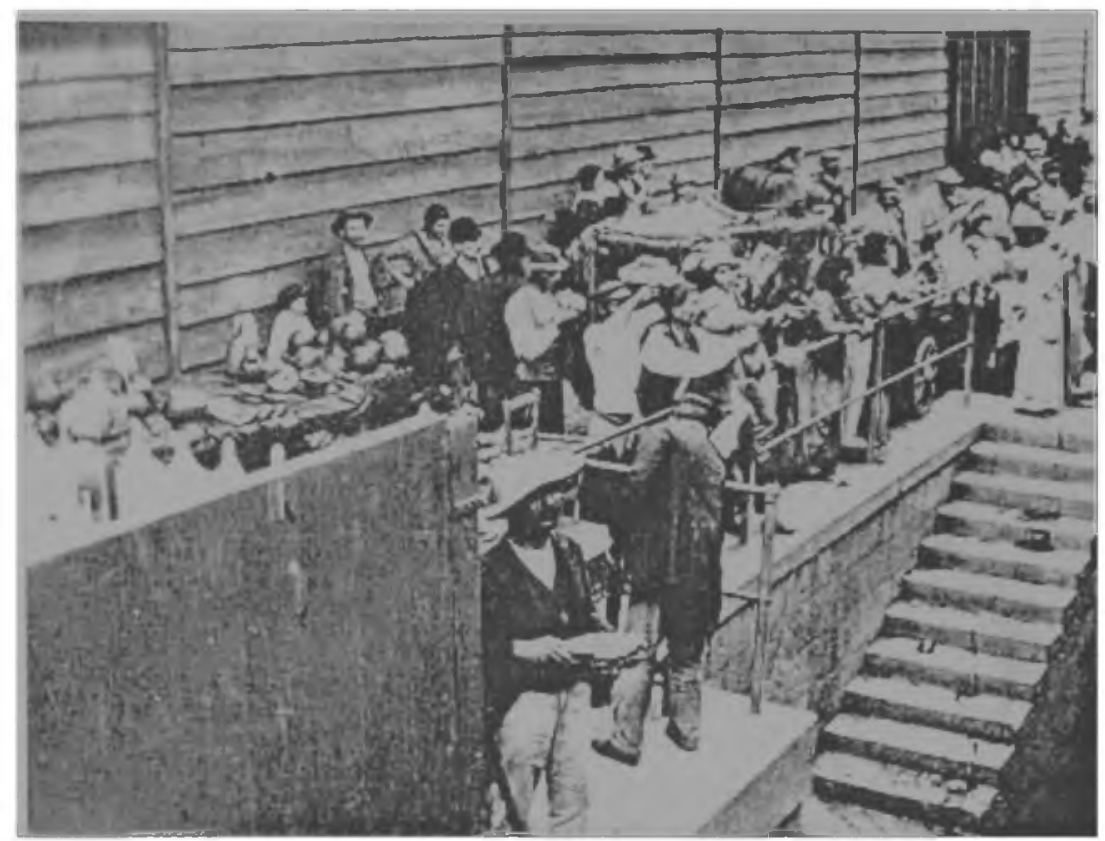

Molo del porto di Genova, inizi Novecento

La fotografia faceva parte della mostra Tante Patrie una Patria. L'identità italiana nel mondo attraverso l'emigrazione, il cui catalogo è stato curato da Stolfi Ėmanuele, Italiani nel Mondo, Mondadori, Milano, 2003, p. 56.

Il bastimento si allontanava e i fili di lana restavano stretti fra le mani degli emigranti a bordo e dei parenti a terra, fino a quando non si rompevano: si trattava di un gesto rituale con una valenza simbolica paradigmatica. Gli emigranti, molto spesso, avrebbero passato la vita intera nel tentativo di riallacciare i capi di quei fili, quei legami familiari, identitari e comunitari che si erano spezzati alla partenza dal porto. Le lettere sono proprio la testimonianza di questo sforzo, di questo compito impossibile: la ricerca costante di ricostruire, o mantenere immutato, attraverso la scrittura, ciò che l'emigrazione aveva irrimediabilmente interrotto o modificato.

Per quanto riguarda in particolare gli aspetti linguistici del fenomeno, che sono il nodo centrale di questo nostro breve articolo, innanzi tutto occorre chiarire un punto: il binomio emigrante- 
analfabeta, spesso considerato una condizione magari non assoluta, ma di certo largamente maggioritaria, andrebbe analizzato alla luce delle relazioni che si instaurano fra mobilità geografica, modernizzazione, sviluppo dell'apparato burocratico dello stato e alfabetizzazione. ${ }^{1}$ Quanto e come l'emigrazione abbia influito sulla diffusione delle pratiche di scrittura fra le classi subalterne è un fatto noto e riconosciuto fin dal classico studio, del 1963, di Tullio de Mauro:

L'efficacia linguistica dell'emigrazione italiana verso l'estero è stata valutata finora andando in traccia degli italianismi lessicali diffusi in altri paesi dagli emigranti e degli esotismi lessicali introdotti dai medesimi in Italia (specialmente nella Lucchesia che sin dal 1870-80 fu una delle zone di massime punte emigratorie). Poiché gli uni e gli altri sono ben poca cosa, si può essere indotti a sottovalutare l'importanza linguistica dell'emigrazione; in realtà questa ha agito sulla situazione linguistica italiana in modo più complesso e profondo di quanto non sia possibile scorgere catalogando gli esotismi introdotti per via popolare in qualche regione italiana. ${ }^{2}$

De Mauro proseguiva nel suo argomentare illustrando gli effetti dell'emigrazione sulla diffusione dell'alfabetizzazione e, di conseguenza, di una lingua comune a discapito della cultura dialettale, legata alla tradizione orale. Con particolare riferimento alla storia della cultura scritta viene ugualmente messo in evidenza il nesso emigrazione e pratica della scrittura da Attilio Bartoli Langeli, in un suo recente lavoro, in cui non esita e definire la Grande emigrazione, insieme alla Grande Guerra, come

le due esperienze di massa [...] le quali causarono una produzione scritta di massa [...] È come se gli emigranti e i soldati abbiano attinto, subitaneamente e massicciamente, alle potenzialità di scrittura depositate nel fondo della società italiana dalla sua lunga e tortuosa storia di rapporto con l'alfabeto. ${ }^{3}$

Ed è proprio la scrittura epistolare che si consacra come il terreno per eccellenza delle pratiche scrittorie delle classi subalterne. Armando Petrucci, uno dei più autorevoli studiosi di storia della scrittura, ha dedicato il suo più recente studio proprioall'epistolografiae, riferendosialla corrispondenza di guerra - ma si potrebbe estendere la valutazione anche a quella di emigrazione - ha affermato:

Quella che può essere a giusta ragione definita l'epistolarità bellica dei subalterni costituisce nel Novecento occidentale il «fiume carsico», cioè sotterraneo e perciò poco conosciuto o misconosciuto, dell'epistolarità contemporanea, anche per le sue diffuse e qualificanti caratteristiche, ovunque presenti, non soltanto di approssimazione (o di invenzione) linguistica, ma pure, se non soprattutto, di diversità materiale e grafica

1. Per le dovute argomentazioni e gli opportuni riferimenti bibliografici a questo proposito rimando a quanto ho già scritto in $M o d e r n i z z a z i o n e$ e pratiche comunicative. La scrittura dell'italiano nel XX secolo, in uItalianística», n. XII, in particolare pp. $115-132$.

2. DE MAURO Tullio, Storia linguistica dell'Italia unita, Laterza, Roma-Bari $2005^{9}$, pp. 54-55. Sempre riguardo all'approccio linguistico, la questione lingua italiana/emigrazione - con un primo bilancio bibliografico sull'argomento - è stata recentemente affrontata in maniera più ampia ed approfondita da VEDOVELLI Massimo, L'italiano degli stranieri, Carocci, Roma 2002, in particolare pp. 111-164; anche se non viene fatto nessun riferimento specifico alla questione della scrittura della lingua e delle pratiche comunicative.

3. BARTOLI LANGELI Attilio, La scrittura dell'italiano, Il Mulino Bologna 2000, p. 156. 
rispetto alla produzione epistolare borghese: matita (spesso copiativa) invece della penna stilografica o della macchina per scrivere; cartolina postale, spesso illustrata (il primo rapporto con il o la corrispondente era la scelta dell'immagine), invece di vere e proprie lettere scritte su fogli ripiegati e imbustati; disordine immaginativo con frequente ricorso a scrittura nei margini; assenza o eccezionalità di punteggiatura; ripetizione maldestra di più o meno antichi modelli scolastici o addirittura incerta imitazione di modelli burocratici o manualistici. ${ }^{4}$

E le osservazioni di Petrucci ci rimandano immediatamente dalla questione materiale del rapporto col mezzo scrittorio, a quella linguistica: ossia in quale lingua o varietà linguistica scrivevano i nostri emigranti? È evidente che gli scriventi avevano ben poca dimestichezza con carta, penna e calamaio, per cuil'esercizio della scrittura continuerà ad essere un'impresa oltremodo faticosa, quasi uno sforzo contro natura, da cui il paradosso delle lettere di illetterati - secondo la definizione di Filippo Lussan $\mathrm{a}^{5}$ - che presentano caratteristiche generali comuni: persistenza di registri espressivi tipici dell'oralità, incerta distinzione tra l'uso di lettere maiuscole e minuscole, difficoltà di separare correttamente le parole e nell'utilizzo dei segni d'interpunzione. Il primo ad interessarsi dal punto di vista linguistico a questi documenti è stato Leo Spitzer, filologo e italianista austriaco che - ironia della storia - venne preposto all'ufficio della censura militare con il compito di controllare la posta dei prigionieri di guerra italiani durante la prima guerra mondiale. Il suo studio ${ }^{6}$ del 1921 è diventato il capostipite degli studi linguistici sull'italiano popolare, termine che ancor oggi suscita discussioni. Si tratta insomma di quella varietà linguistica che è stata definita dagli specialisti italiano popolare o semicolto. ${ }^{7}$ Ovvero, l'italiano appreso in modo non completo e non corretto da chi possiede come lingua madre il dialetto. Una lingua piena di termini regionali e con proprie particolarità, di cui sono stati identificati 28 tratti costanti che la caratterizzano come varietà linguistica compiuta. $\mathrm{Ma}$, al di la delle definizioni, siamo di fronte al risultato di un enorme sforzo collettivo di comunicazione che, non potendo trovare altra via d'uscita se non quella dell'italiano, segna cosi una prima importante tappa verso il raggiungimento di un'identità linguistica nazionale. Ma non solo, per riprendere ancora una volta le osservazioni fatte sul lungo periodo da Bartoli Langeli:

l'italiano popolare è un modo di scrivere, non di parlare; e ha un carattere largamente unitario, sovraregionale. Gli specialisti vi identificano si tratti dialettali e locali, ma in numero e con incidenza relativamente bassi. Soltanto i letterati sono in grado di scrivere il dialetto. Gli illetterati, per il solo fatto di aver imparato a scrivere, realizzano quella che essi ritengono la lingua scrivibile, non la lingua che parlano. Molti dei fenomeni riscontrabili nei loro testi non hanno, non possono avere un'effettiva corrispondenza di pronuncia. ${ }^{8}$

4. PETRUCCI Armando, Scrivere lettere. Una storia plurimillenaria, Laterza, Roma-Bari 2008, p. 186.

5. LUSSANA Filippo, Lettere di illetterati. Note di psicologia sociale, Zanichelli, Bologna s.d. [1913].

6. SPITZER Leo, Lettere di prigionieri di guerra italiani 1915-1918, Boringhieri, Torino 1976.

7. Cfr. DE MAURO Tullio, Storia linguistica dell'Italia unita, cit.; CORTELLAZZO Manlio, Avviamento critico allo studio della dialettologia italiana, Vol. III, Lineamenti di italiano popolare, Pacini, Pisa 1972; COVERI Lorenzo, Italiano popolare, scrittura popolare: una prospettiva linguistica, in «Materiali di lavoro», n. 1-2, 1987; BERRUTO Gaetano, L'Italiano popolare, in IDEM, Lineamentidi sociolinguistica dell'italiano contemporaneo, La Nuova Italia Scientifica, Roma 1987, pp. 107-140; BRUNI Francesco (a cura di), L'italiano nelle regioni. Lingua nazionale e identità regionali, Utet, Torino 1992.

8. BARTOLI LANGELI Attilio, La scrittura..., op. cit., p. 168. 
Ed è un modo di scrivere che ha degli elementi permanenti che costituiscono una lunga durata - la definizione è ancora di Bartoli Langeli - in cui è possibile rintracciare caratteristiche comuni tali da poter affermare che lo scrivere dell'italiano popolare è

il modo medievale di scrivere l'italiano. A leggere gli autografi "popolari' ottonovecenteschi hai l'impressione che poco o nulla sia cambiato dai tempi iniziali della diffusione dello scrivere volgare, che i seicento anni che separano il Ghezzo fattore che scrive nel 1314 al suo principale dai soldati che scrivono alle famiglie dal fronte $o$ dai campi di prigionia siano una linea continua. Dal nostro punto di vista beninteso, nel senso cioè che su questi scriventi la svolta cinquecentesca (la codificazione grammaticale e ortografica dell'italiano) e gli avanzamenti dell'alfabetizzazione formale non hanno prodotto grandi effetti. Quel modo di scrivere, inizialmente largo e libero per l'assenza di una norma, si è mantenuto a lungo anche dopo che l'italiano fu ridotto a norma, fuori e contro di essa. ${ }^{9}$

Viene così individuata una linea di continuità che attraversa i secoli, che ha rappresentato una produzione sorda e sommersa di comunicazione scritta, che si è sviluppata in una sorta di zona grigia, al confine fra oralità e scrittura, o meglio, fra cultura orale e cultura scritta, i cui autori sono uomini e donne delle classi subalterne che hanno impugnato la penna pur senza dominarla completamente ed hanno - nella maggior parte dei casi, inconsapevolmente - esercitato il proprio diritto alla scrittura sfidando una società che del privilegio e dell'esclusività di questo diritto ne faceva una discriminante per l'esclusione. Che questa produzione scrittoria venisse trasformata in un vero e proprio mare di carta nel corso del fenomeno migratorio proprio da coloro i quali si ritrovavano nelle condizioni di esercitare un al tro diritto che per definizione si colloca anch'esso in una zona grigia e di confine, il diritto alla mobilità 0 , come è stato definito, alla fuga,$^{10}$ rafforza ancor più la suggestiva immagine dell'irriducibilità di questa produzione scritta ai canoni della cultura alta o comunque colta, nonché la sua vivacità e vitalità.

\title{
Le lettere di chiamata tra documento intimo e formulario burocratico
}

\author{
«Inspetoria de Immigração do Estado de São Paulo no Porto de Santos» \\ Certifico que o senhor Carlo D'Abruzzo, de nacionalidade italiana, com mais de 60 \\ annos de edade, casado, que se acha actualmente em Sant'Angelo de Pesco, (Provincia \\ de Campobasso, Italia) e deseja viajar para o Brasil, é tio do senhor pascoal de Palatis
}

9. BARTOLI LANGELI Attilio La scrittura..., op. cit., pp. 166-167.

10. MEZZADRA Sandro, Diritto di fuga. Migrazioni, cittadinanza e globalizzazione, Ombre Corte, Verona 2006. Occorre specificare però che esercitare il diritto alla scrittura non si può identificare direttamente e meccanicamente con un atto emancipatore; anzi, almeno fino al secondo dopoguerra e soprattutto in contesto bellico, la scrittura e la diffusione della pratica della comunicazione scritta fra le classi subalterne hanno rappresentato un volano, una cinghia di trasmissione delle parole d'ordine, degli slogan della propaganda e dei valori del potere e dello stato, fosse quello liberale o il regime fascista; cfr. GIBELLI Antonio, L'officina della guerra, La Grande Guerra ele trasformazioni del mondo mentale, Bollati Boringhieri, Torino $1998^{2}$, pp. 99-102; CROCI Federico, Scrivere per non morire. Lettere dalla Grande Guerra del soldato bresciano Francesco Ferrari, Marietti (ora Paravia-Scriptorium) Genova 1992, pp. 64-\{,6. GIBELLI Antonio, Il popolo bambino. Infanzia e nazione dalla Grande Guerra a Salò, Einaudi, Torino 2005, pp. 277-290. Il fenomeno assume dimensioni macroscopiche in situazioni di di- 
morador na estação de Ribeirão Pires (município de S. Bernardo, Estado de S. Paulo, Brasil) onde exerce a profissão de canteiro e é proprietário da pedreira denominada São Caetaninho, em cuja companhia e a cujas expensas passará a viver o deu tio acime referido quando vier para o Brasil, não avendo, porisso, nenhum impedimento para o desembarque do mesmo no porto de Santos, uma vez que não soffra de molestia contagiosa e exhiba o presente certificado ás autoridades que comparecerem nо acto do céu desembarque.

Santos, 13 de outubro de 1921 [Timbro d'imbarco nel porto di Napoli del 24 aprile 1922] [Timbro di sbarco nel porto di Santos del 24 maggio 1922] ${ }^{11}$ I s an ciamè (ci hanno chiamato) dalla testimonianza di Enrica Cesari, 20012

Sull'epistolografia d'emigrazione esiste ormai una letteratura consolidata da circa trent'anni di studi, ${ }_{3}^{13}$ ma in questo caso specifico ci occupiamo di Lettere di Chiamata, ossia di quelle pratiche che oggi, nei termini della burocrazia delle migrazioni, verrebbero definite di ricongiungimento familiare. In sostanza di cosa si tratta? Le Lettere di Chiamata si possono suddividere in due grandi gruppi che fanno riferimento alla diversa tipologia dei documenti: un primo e più consistente gruppo è quello che chiameremo delle Lettere Ufficiali, costituito da documenti redatti su appositi moduli presso i consolati dei paesi interessati nello Stato di São Paulo, o presso gli uffici addetti della Inspectoria de Imigração del porto di Santos, come nel caso del documento citato in epigrafe in questo paragrafo; il secondo gruppo è formato da delle classiche Lettere Private, ossia lettere manoscritte, corrispondenza privata, ricevuta dal Brasile che il parente in partenza dall'Italia portava con sé a testimonianza del fatto che andava laddove c'era qualcuno che lo potesse ricevere e mantenere, non necessariamente si trattava di lettere nelle quali si faceva un seppur informale atto di chiamata.

In particolare per quanto riguarda l'immigrazione italiana nello Stato di São Paulo, il maggior flusso d'entrata di cittadini italiani si è registrato negli anni compresi tra il 1887 e il 1902, ${ }^{14}$ ingressi

slivello di potere tra mittenti e destinatari della corrispondenza, cfr. ZADRA Camillo e FAIT Gianluigi (a cura di), Deferenza. Rivendicazione. Supplica. Lettere ai potenti, Treviso, Pagus Edizioni, 1991.

11. Lettera di chiamata, Arquivo do Memorial do Imigrante (AMI), São Paulo.

12. Citato in SERVETTI Lorenza, Vado nella Merica; è lì di là dalle colline. Budrio e la grande emigrazione (1880-1912), Marsilio, Venezia 2003, p. 21, che prosegue specificando che «cosi veniva risposto a Enrica Cesari quando chiedeva ai suoi genitori perché, tanti anni prima, fosseropartiti per il Brasile; equesta frase rimase per leialungo misteriosam. La testimonianza è stata rilasciata all'autrice l'8 giugno del 2001 dalla figlia di Anselmo Cesari e Maria Treggia che sono partiti per il Brasile nel 1895 e ritornati a Budrio circa dieci anni dopo.

13. Sulle scritture degli emigranti e sulle loro potenzialità come fonti per la storia, si potrebbero citare i classici di THOMAS William Isaac e ZNANIECKI Florian sulle lettere di emigranti polacchi risalente agli anni 1918-1920 (cfr. Il contadino polacco in Europa e in America, Edizioni Comunità, Milano 1968, 2 voll. [ed. or. The University of Chicago Press 1918-1920]). Limitandosi al caso italiano, il riferimento d'obbligo va al pionieristico lavoro di FRANZINA Emilio, Merica! Merica Emigrazione e colonizzazione nelle lettere dei contadini veneti efriulani in America Latina (1876-1902), Milano, Feltrinelli, 1979 (Verona, Cierre Edizioni, 1994²), seguito dagli studi di CHEDA Giorgio sui ticinesi in Australia e California (cfr. L'emigrazione Ticinese in Australia, Armando Dadò Editore, Locarno 1979; L'emigrazione Ticinese in California, Armando Dadò Editore, Locarno, 1981). Ma per limitarci agli studi piủ recenti - cui rimandiamo anche per ulteriori riferimenti bibliografici - che fanno il punto della situazione sia rispetto al percorso storiografico, sia sui principali elementi dell'epistolografja popolare d'emigrazione, come i contenuti prevalenti della corrispondenza, il dettaglio delle possibili funzioni, nonché alcune fondamentali indicazioni di carattere metodologico, si vedano GIBELLI Antonio e CAFFARENA Fabio, Le lettere degli emigranti, in BEVILACQUA Piero a, DE CLEMENTI Andreina e FRANZINA Emilio (a cura di), Storia dell'emigrazione italiana, Vol. I, Partenze, Roma, Donzelli, 2001, pp. 563-574.

14. Mi riferisco a flussi superiori alle 30.000 unità all'anno, con picchi di 105.000 unità nel $1888,131.000$ nel $1891,98.000$ nel 1895 , 105.000 nel 1897, 60.000 nel 1901. Dal 1902, l'anno del decreto Prinetti (provvedimento che, in seguito alle denunce delle condizioni in cui erano costretti a lavorare i braccianti italiani nelle fazendas di caffè arrivati con il viaggio pre-pagato, ha bloccato l'immigrazione sussidiata), gli ingressi annuali rimarranno sempre al di sotto dei 28.027 (quota fissata come limite massimo dell'immigrazione annuale) eccezion fatta per gli anni 
che comunque avevano un saldo positivo, ossia in relazione alle uscite, di circa il $40 \%$. Una parte considerevole del movimento nei primi anni del XX secolo si deve all'effetto chiamata, vale a dire a quello che, fin dalle classiche formulazioni delle catene migratorie, ${ }^{15}$ è il ruolo attribuito ai primi arrivatiche, soprattutto attraverso la corrispondenza privata, richiamano o invitano a raggiungerli parenti, amici e conoscenti. Sono coloro i quali risultano nelle statistiche dei flussi a cavallo tra il XIX e il XX secolo come saldo positivo, in altre parole quelli che restano e non ritornano a casa, che costituiscono una base d'appoggio e uno dei fattori d'attrazione dei flussi migratori che seguono, $o$ meglio sarebbe dire costituiscono uno dei nodi attraverso i quali funzionano le reti migratorie. $\mathrm{E}$ le lettere sono la trama di queste reti, il canale di comunicazione, la linfa vitale che le sostiene.

Dal punto di vista istituzionale, nel 1911, è intervenuta una legge brasiliana che in un certo senso legalizzava l'uso della Lettera di Chiamata. Ad esempio, introduceva l'obbligo della chiamata per i maggiori di 60 anni e i non abili al lavoro:

\section{Parágrafo único. Os maiores de 60 anos e os inaptos para o trabalho só serão admitidos quando acompanhados de suas famílias, ou quando vierem para a companhia destas, contanto que haja da mesma família, pelo menos, um individuo valido, para outro invalido ou para um até dois maiores 60 anos.}

L'unico modo per dimostrare che il migrante arrivava per stare in famiglia e questa era disponibile e atta al loro mantenimento era munirsi di una Lettera di Chiamata. Nel successivo Capitolo II $\operatorname{art.} 5^{\circ}$, si specificava che il Governo Federale avrebbe fornito gratuitamente agli agricoltori che fossero arrivati con famiglia «ou chamados das mesmas», sempre che abili al lavoro, il biglietto di terza classe, trasporto e accoglienza fino alla destinazione scelta, esenzione dal pagamento delle tasse per i bagagli e gli attrezzi agricoli o utili all'esercizio della professione dichiarata: un ottimo motivo per presentarsi con una Lettera di Chiamata. E ancora nel Capitolo III art.

1912 e 1913 in cui vi furono rispettivamente 31.000 e 30.000 ingressi, cfr. AESP (Arquivo do Estado de São Paulo), serie Inspetoria de Terras e Colonização, Boletim da Diretoria de Terras, Colonização, e Imigração, n. 1, 1937.

15. Com’è noto, per catena migratoria s'intende il modello che consente lo studio dei meccanismi che favoriscono o inibiscono l'arrivo degli immigranti o la partenza degli emigranti - secondo il punto di vista - esortati e consigliati da parenti, amici o compaesani, nel caso della cosiddetta catena informale, familiare o comunitaria; nel caso della catena formale o istituzionale i meccanismi sono messi in atto dalla stampa, la pubblicità o la propaganda fatta da agenti contrattati dalle compagnie di navigazione, o dalle agenzie delle società d'immigrazione che i governi dei paesi americani finanziavano. L'elaborazione del concetto di catena migratoria rimonta alla sociologia degli anni Cinquanta del XX secolo, anche se, in realtà, è stata una categoria che la storiografia italiana ha sottovalutato. Cionondimeno, dagli anni Sessanta ha contribuito al cambiamento di prospettiva degli studi migratori, valorizzando l'uso dei documenti personali nell'indagine e interpretazione delle dinamiche dell'esodo. Oggi ormai possiamo contare su di una vasta letteratura; riguardo all'emigrazione italiana in America Latina, forse il caso argentino è stato l'ambito in cui si sono approfondite di più le ricerche. Per un bilancio di questi lavori e le linee essenziali del dibattito si veda il numero monografico di «Estudios Migratorios Latinoamericanos», Las cadenas migratorias italianas a la Argentina, n.8 1988. Come ormai è stato ampiamente dimostrato il modello delle catene ha presentato problemi di ambiguità e rigidità, che sono impliciti nell'artificiosa linearità che la stessa immagine della catena impone. Le ricerche più recenti hanno invece posto l'accento sulla varietà e variabilità dei movimenti migratori e, soprattutto, sulla molteplicità delle destinazioni, intrecciate a tal punto che, come ha suggerito Fernando DEVOTO, è ormai diventato necessario sostituire la metafora della catena con quella della ragnatela, cfr. DEVOTO Fernando, Algo más sobre las cadenas migratorias de los italianos a la Argentina, in «Estudios Migratorios Latinoamericanos», n.19, 1991, p. 342; Ídem, Le migrazioni italiane in Argentina. Un saggio interpretativo, Officina Tipografica, Napoli 1994, p. 48. Tra i lavori recenti di Devoto, oltre allo studio comparato su Argentina e Brasile svolto con Boris Fausto (cfr. FAUSTO Boris e DEVOTO Fernando, Brasile Argentina: um ensaio de história comparada (1850-2002), Editora 34, São Paulo 2005'), occorre ancora citare per l'interessante approccio "globale" il volume Historia de la Inmigración en la Argentina, Editorial Sudamericana, Buenos Aires 2003; e il volume appena uscito per i tipi di Donzelli, Storia degli italiani in Argentina, Roma 2007. Dalle catene, alle ragnatele, per arrivare a un modello che negli ultimi anni ha acquisito una sempre maggior solidità grazie anche alla moltiplicazione degli studi che ne hanno fatto uso: le reti. Lo stesso DEVOTO nel 1994 ne faceva già uso in Liguri nell'America australe: reti sociali, immagini, identità, in RUGAFIORI Paride e GIBELLI Antonio (a cura di), Storia d'Italia, Le regioni, dall'unità a oggi. La Liguria, Einaudi, Torino 1994, pp. $651-688$. A questo proposito si veda anche RAMELLA Franco, Reti sociali, famiglie e strategie migratorie, in BEVILACQUA Piero, DE CLEMENTI Andreina, FRANZINA Emilio (a cura di), Storia dell'emigrazione italiana, Vol. I, Partenze, Donzelli, Roma 2001, pp. 143-160. 
$18^{\circ}$ si dava preferenza, per l'imbarco con le compagnie di navigazione autorizzate dal governo federale al trasporto dei migranti, ai cosiddetti immigranti spontanei e a quelli «chamados por parentes já estabelecidos no Brasil».16

Tanto per quantificare il fenomeno, si tratta di un movimento che ha coinvolto, dal 1900 al 1929 , più di 350.000 persone, ${ }^{17}$ proprio nella fase in cui la regione paulista stava vivendo un processo di profonda trasformazione della struttura produttiva e dell'organizzazione sociale. Fino ad oggi ci si è basati sulle statistiche che sono state pubblicate nei bollettini della Secretaria de Agricoltura, senza avere la possibilità di consultare le carte originali. ${ }^{18}$ Una parte degli originali delle Lettere di Chiamata è custodito presso l'archivio del Memorial do Imigrante, sede dell'antica Hospedaria di São Paulo. ${ }^{19}$ Gli immigranti che sbarcavano nel porto di Santos consegnavano la Lettera di Chiamata ai funzionari dell'Inspectoria de Imigração che, a loro volta, le depositavano, insieme alle liste di sbarco dei piroscafi e ad altra documentazione, all'Hospedaria.

Di Lettere Ufficiali, ossia i documenti formali che venivano usati per farsi autorizzare lo sbarco nel porto di Santos, ve ne sono di diverso tipo: quelli redatti presso il Consolato Generale d'Italia a São Paulo (o presso altri consolati, secondo la nazionalità dello scrivente) su moduli prestampati, dai quali si possono desumere i dati anagrafici fondamentali e la professione sia del richiamante che del richiamato; quelli redatti dalla stessa Inspectoria de Imigração del porto di Santos su richiesta del richiamante, che svolgono la funzione di nulla osta allo sbarco, quindi redatti a Santos, spediti in Italia e ritornati a Santos in mano al richiamato; quelli redatti dai Comuni in Italia con diverse tipologie, ma quasi sempre in funzione di nulla osta allo sbarco.

Molti dei documenti, ad esempio, più che dei veri e propri ricongiungimenti familiari attestano una mobilità continua tra i due paesi (e non di rado la rete include punti e snodi in altri paesi, spesso, ad esempio l'Argentina) che queste reti familiari hanno mantenuto per molti anni:

Il Sindaco Certifica che Franchi Alessandro fu Giuseppe, nato in Careggine, residente in Castelnuovo, da oltre quaranta anni residente in San Paolo, Brasile dove possiede beni stabili e commerci, e la propria famiglia, che colà lo chiama avendo urgenza di fare liquidazioni e vendite, essendo urgente la sua presenza per eseguire dette operazioni. Quindi il detto Franchi Alessandro deve nuovamente espatriare d'urgenza per il Brasile

Il Sindaco di Castelnuovo di Garfagnana, 12 giugno $1922^{20}$

16. Cfr. Decreto n. 9.081 de 03 de novembro de 1911, Dá novo regulamento ao Serviço do Povoamento, in HORN IOTTI Luiza (a cura di), Imigração e colonização, legislação 1747 a 1915, Educs, Caxias do Sul 2001, p. 522-572. La legge è suddivisa in 26 Capitoli per un totale di 277 articoli; il capitolo XXIII che comprende gli articoli dal n. 231 al n. 238, tratta dell'Hospedaria da Ilha das Flores, quella del porto di Rio de Janeiro, dettagliando le norme per l'entrata, la permanenza e l'uscita degli immigranti dall' Hospedaria, l'unica che era finanziata e riconosciuta dal Governo Federale.

17. Cfr. AESP, serie Secretaria da Agricultura, Departamento de Imigração e Colonização, Estatística dos trabalhos executados pelo Departamento de Imigraçāo e Colonização durante o ano de 1961, São Paulo, 1962, p.44.

18. Cfr. Cfr. VANGELISTA Chiara, Os Braços da lavoura. Imigrantes e caipiras na formação do mercado de trabalho paulista (1850-1930), São Paulo, HUCITEC 1991, pp. 96-114.

19. Le Lettere di Chiamata ad oggi consultabili, suddivise in 5 casse, sono circa 2.000 per un periodo compreso tra il 1911 e il 1927 , approfitto dell'occasione per ringraziare la direttrice esecutiva del Memorial do Imigrante, dona ANA MARIA LEITÃO VIEIRA per avermi dato la più completa disponibilità e libertà di consul tazione dell'Archivio, un ringraziamento particolare anche dona MIDORY KIMURA FIGUTI, che mi ha svelato ogni segreto dell'archivio del Memorial, da lei curato con rara passione e competenza da oltre trent'anni. Oggi le Lettere di Chiamata sono oggetto di un progetto di ricerca del LEER-USP (Laboratorio de Estudos sobre Etnicidade Racismo e Discriminação), sviluppato in compartecipazione con il Memorial do Imigrante, l'Università di Genova, l'Archivio di Stato di São Paulo, l'Istituto Italiano di Cultura e l'Istituto Cultural Ítalo-Brasileiro, per la costituzione di un Arquivo Virtual da Imigraçāo Italiana.

20. Lettera di chiamata, AMI, São Paulo. 
In questo caso - e di certificati simili rilasciati dai comuni se ne trovano molti fra le lettere di chiamata - è evidente che questa specie di "autochiamata" è una sorta di attestato di relazioni, non solo affettive e familiari, ma anche economiche che si sono mantenute per decenni tra una parte della famiglia emigrata in Brasile e l'altra parte che è rimasta in Italia.

\section{"Saressimo desiderosi di sapere"}

E più che altro mi attiravano i sacchi della posta, accumulati in un canto, legati e suggellati. Poiché v'eran là dentro i frammenti del dialogo di due mondi: chi sa quante lettere di donne che per la terza o quarta volta chiedevano dolorosamente notizie del figliuolo o del marito, che non si facevan vivi da anni; e supplicazioni perché tornassero o le chiamassero a raggiungerli; domande di soccorso, annunzi di malattie, e di morti; e ritratti di ragazzi che i padri non avrebbero più riconosciuti, e richiami desolati di fidanzate e menzogne impudenti di mogli infedeli e ultimi consigli di vecchi: tutto questo mescolato a letteroni irti di cifre di banchieri, a espistole amorose di ballerine e di coriste [...]. E tutti quei sacchi si sarebbero sparpagliati fra pochi giorni dalle foci del Plata ai confini del Brasile e della Bolivia e fino alle rive del Pacifico e nell'interno del Paraguay e su per i fianchi delle Ande, a suscitare allegrezze, rimorsi, dolori, timori; i quali poi a volta loro, pigiati in altri sacchi, avrebbero fatto in direzione opposta il medesimo viaggio, ammucchiati in un altro camerino come quello, dove avrebbero visto passare altre processioni di povere genti, che se ne ritornavano al mondo vecchio, forse meno poveri, ma non più felici di quando l'avevano abbandonato con la speranza d'una sorte migliore.

Edmondo De Amicis, Sull'Oceano, 188921

Tencionando a Diretoria do Serviço de Propaganda e Exportação Econômica do Brasil no Estrangeiro redigir um opúsculo, salientando as condições favoráveis de que gozam os colonos estabelecidos no Brasil [...] solicito que sejam remetidas cartas de colonos, dirigidas a parentes seus na Europa e nas quais eles façam alusão á sua satisfação por haverem emigrado para aqui[...] ao melhoramento que obtiveram nas suas condições econômicas.

Comunicado de 13 de fevereiro de $1909^{22}$

La lontananza, abbiamo detto, è l'impulso determinante per scatenare la scrittura e, in contesto migratorio, assurge a un significato peculiare ed emblematico, fino ad evocare una condizione

21. DE AMICIS Edmondo, Sull'Oceano, (a cura di BERTONE Giorgio), Diabasis, Reggio Emilia, 2005, pp. $210-211$. 22. Cfr. AESP (Arquivo do Estado de São Paulo), serie Secretaria da Agricultura 1823-1926, Comércio e Obras Públicas, Comunicado de 13 de fevereiro de 1909. 
esistenziale per gli emigranti. Dall'iniziale lontananza fisica, geografica, affettiva, che viene dolorosamente percepita già dalla partenza, momento della genesi della condizione migrante, fino alla lontananza che si alimenta del dilatarsi dello spazio e del tempo. Nonostante il paziente e tenace lavoro di scrittura nel tentativo di ricucire lo strappo della separazione, inevitabilmente, con il suo prolungarsi da situazione temporanea sempre rivolta a un possibile pronto ritorno, la lontananza iniziale muta in condizione permanente e genera lontananza culturale, linguistica e identitaria. ${ }^{23}$ La lettera si configura dunque come il paradigma dell'emigrazione: frutto dell'allontanamento e della separazione, prodotto del bisogno di comunicazione a distanza, risultato del tentativo di annullare le distanze e, al tempo stesso, testimonianza e prova della lacerazione in corso nonché veicolo delle trasformazioni future.

In merito ai contenuti, le lettere hanno permesso di indagare l'integrazione degli emigrati nel paese d'accoglienza, la disgregazione familiare, l'allontanamento e la ricongiunzione, la persistenza o il mutamento degli atteggiamenti mentali tradizionali, i conflitti di classe e le risposte delle diverse generazioni alle sfide che le nuove società proponevano loro. Proprio l'utilizzo di fonti quali lettere, diari, memorie e fotografie degli emigranti, ha permesso di dare al fenomeno emigrazione una profondità maggiore, andando tra le pieghe di scelte e motivazioni spesso anche molto diverse tra loro, restituendoci i sogni e le speranze di tante persone comuni che hanno solcato l'Oceano per raggiungere le Americhe, insieme alle dinamiche soggettive ma anche alle reti relazionali tra comunità di partenza e di arrivo che si articolavano all'interno dei flussi migratori.

In effetti, attraverso le missive non si mantengono semplicemente i contatti con i familiari, ma si forniscono importanti elementi che determinano le scelte di chi deve ancora prendere in considerazione l'opportunità di partire o è già in procinto di farlo: in particolare le mete d'arrivo sono valutate in relazione alle possibilità di lavoro offerte e chi è emigrato getta le basi per la costruzione di un ponte di notizie. Per questo le missive hanno una doppia valenza: da una parte sono testi domestici ad uso familiare, dall'altro assumono anche una rilevanza pubblica in quanto si rivelano insostituibili fonti informative di prima mano, sulle quali si ripone una fiducia non concessa alle statistiche ufficiali o alle lusinghe degli agenti d'emigrazione. L'appello della Secretaria de Agricoltura, che abbiamo citato all'inizio del paragrafo, mirava proprio a fare un uso pubblico delle lettere dei coloni ai parenti per dare maggior impulso alla propaganda pro-emigrazione sviluppata dalle agenzie del governo all'estero. La stampa dell'epoca in Italia, sia pro o anti emigrazionista, fece largo uso della corrispondenza privata nell'intento di render più credibile la propria linea politica. Le reti migratorie che svuotano interi paesi hanno quindi delle resistenti maglie di carta che regolano ed indirizzano il flusso dei partenti. ${ }^{24}$

23. Mi permetto qui di rimandare a CROCI Federico e BONFIGLIO Giovanni, El baúl de la memoria. Testimonios escritos de inmigrantes italianos en el Perú, Fondo Editorial del Congreso de la República del Perú, Lima 2002; in particolare al primo capitolo Palabras de la lejanía. Escritos de inmigrantes como fuente histórica, pp. 19-35.

24. Sulle scritture degli emigranti e sulle loro potenzialità come fonti per la storia, e per la storia della lingua in Brasile non si è ancora fatta strada una solida tradizione di studi. Esistono molte pubblicazioni, soprattutto di testimonianze autobiografiche nella maggior parte dei casi riferite all'esperienza della colonizzazione degli stati del Sud la cui edizione si deve alla cura e al lavoro di studiosi come Luis Alberto DE BONI e Rovílio COSTA, per gli opportuni riferimenti bibliografici rimando a FRANZINA Emilio, L'immaginario degli emigranti. Miti e raffigurazioni dell'esperienza italiana all'estero fra i due secoli, Pagus, Treviso 1992. Per quanto riguarda l'uso delle testimonianze scritte come fonte per la storia, riguardo all'esperienza della colonizzazione agricola, il riferimento d'obbligo va al classico, DAVATZ Thomas, Memórias de um colono no Brasil: 1850, Edusp, São Paulo 1980 (con prefazione e note di Sergio BUARQUE DE HOLLANDA); si tratta del noto diariomemoria del maestro della colonia di Ibicaba che, quando venne pubblicato nel 1858 in Svizzera, suscitò un'ondata di sdegno nell'opinione pubblica, identificando il Brasile come un paese inadatto a ricevere immigranti europei. Riguardo alla corrispondenza dei coloni, si veda BENDOCCHI Alves Débora, Cartas de imigrantes como fonte para o historiador: Rio de Janeiro-Turíngia (1852-1853), in «Revista Brasileira de História», n. 45, 2003, pp. 155-184; che analizza nove lettere di immigrati tedeschi nelle fazendas della provincia di Rio de Janeiro negli anni 1852-1853, pubblicate da riviste tedesche dell'epoca e rinvenute negli archivi di Turingia. Nell’ambito specifico dell'analisi linguistica 
Per quanto riguarda le Lettere di Chiamata dell'Hospedaria, addirittura ci sono casi in cui lo scrivente cerca di dissuadere il parente alla partenza in modo esplicito, ma la presenza della lettera fra le carte dell'Hospedaria ci rivela un comportamento in totale dissonanza con i consigli ricevuti dal familiare, ${ }^{25}$ aprendo cosi uno spiraglio molto interessante sulla lettera come veicolo di notizie e regolatore di flussi. In alcuni casi, rari, si tratta di lettere scritte apposta per consentire l'imbarco dei parenti e quindi prive, o quasi, di quelle caratteristiche di testimonianza scritta che rendono le lettere una fonte insostituibile per lo studio degli aspetti culturali della storia dell'emigrazione. In altri, ci troviamo di fronte ad un frammento d'epistolario che é rimasto impigliato nelle maglie della burocrazia statale che cerca di regolare il movimento delle persone alla stregua di quello delle merci. '̇ superfluo sottolineare come questo tipo di carte ci consenta di entrare nell'analisi delle strategie personali e familiari, nonostante la frammentarietà e il carattere episodico dei documenti a disposizione. Non possiamo, infatti, disporre d'epistolari, ma solo di schegge e frammenti isolati d'epistolari che, in alcuni fortunosi casi, possono esser dei micro-epistolari composti di 2 o 3 lettere magari d'andata e ritorno che il parente, ansioso di ricevere il permesso di sbarco, ha consegnato all'Inspectoria de Imigração del porto di Santos.

Per quanto riguarda la questione della lingua, com’è noto, il binomio lingua e cultura ${ }^{26}$ costituisce un'unità inscindibile al cui interno si possono individuare una serie di relazioni articolate e complesse - la lingua traduce la cultura in senso antropologico, la cultura crea la lingua e viceversa ${ }^{27}$ - che contribuiscono alla definizione, o meglio, demarcazione, identitaria del parlante, possiamo dire - per estrema sintesi - che ogni lingua "si appoggia" e si struttura intorno ad un immaginario e a una cultura di appartenenza. Le lettere rappresentano un italiano scritto sottoposto al contatto più o meno prolungato con la lingua e la cultura del paese ospite, sono la cristallizzazione - nero su bianco è il caso di dire - di interferenze lessicali e morfo-sintattiche che spesso si riscontrano anche nel parlato, ma soprattutto ci offrono l'opportunità di studiare dettagliatamente alcune fasi del contatto tra lingua e cultura d'origine e lingua e cultura ospite, offrendo spunti di riflessione sui

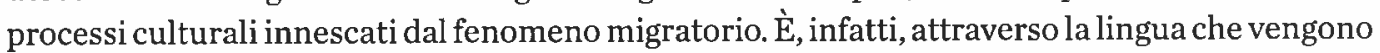
veicolati i processi di acculturazione, integrazione e/o assimilazione con la società ospite. Così come è sulla lingua che vengono applicate o studiate strategie di propaganda e manipolazione del consenso, o di repressione verso comunità considerate potenzialmente pericolose. A questo proposito basti pensare a quanto è avvenuto qui in Brasile durante il periodo dell'Estado Novo con il decreto del 1938 sulla naturalizzazione degli stranieri ed il successivo decreto del 1941 che proibiva la vendita e la circolazione di giornali in lingua straniera e vietava l'uso pubblico di una lingua straniera. ${ }^{28}$ Situazione quasi paradossale in una São Paulo che ancora in quegli anni contava

di lettere di emigranti, per il caso brasiliano si veda BANFI Emanuele, Analisi variazionistiche nelle lettere di un migrante ladino in Brasile a metà '800, in Id. e CORDIN Patrizia (a cura di), Pagine di scuola, di famiglia, di memorie. Per una analisi del multilinguismo nel Trentino austriaco, Museo Storico in Trento, Trento, 1996.

25. Vi é un caso, che si presterebbe a della facile ironia, in cui lo scrivente si rivolge alla suocera pregandola di non andare in Brasile e quasi minacciando di andare lui in Italia o, come seconda soluzione, a Mendoza per ricongiungersi con un'altra parte della famiglia. Insomma avverte la suocera che non vada, altrimenti sarà lui ad andarsene, seguendo i percorsi della "rete" a sua disposizione. Il mittente scrive il 2 febbraio del 1911 e la lettera reca il timbro della Inspectoria de Imigração del porto di Santos del 21 ottobre dello stesso anno.

26. Sulla questione la bibliografia è vastissima, si tratta di uno dei nodi su cui la riflessione antropologica d'inizio Novecento si è interrogata profondamente, mi limito a rimandare al classico di MALINOWSKY Bronislav, Teoria scientifica della cultura, Feltrinelli, Milano 1962; per una panoramica generale si veda FABIETTI Ugo, Storia dell'antropologia, Zanichelli, Bologna 2001, e le riflessioni sviluppate da CARDONA Giorgio Raimondo, Introduzione all'etnolinguistica, Il Mulino, Bologna 1976.

27. Cfr. LONGO di Cristofaro Gioia, MARIOTTI Luciana (a cura di), Modelli culturali e differenza di genere, Armando, Roma 1998 , p. 11.

28. Cfr. MACHADO CAMPOS Cynthia, A politica da língua na era Vargas. Proibição do falar alemão e resistências no Sul do Brasil, UNICAMP, Campinas 2006; BUENO Alexandre Marcelo, Intolerância Lingüística e Imigração, 2006, Tese de Mestrado do Programa de Pós-graduação em Semiótica e Lingüística Geral da FFLCH-USP, orientadora Profa Dra, Diana Luz Pessoa De Barro. 
con una comunità italiana numerosa che si mantenne come il maggior gruppo etnico straniero fino al 1940, anno in cui venne superato dai portoghesi. ${ }^{29}$

Não sei se uma cidade poderia ser mais italiana que São Paulo! No bonde, no teatro, narua, na igreja, falava-se mais o idioma de Dante do que a lingua de Camões. Os maiores e mais numerosos comerciantes e industriais eram italianos [...] Coisa realmente assustadora. A impressão de que íamos perder a nacionalidade, ser absorvidos aterrava. ${ }^{30}$

I regimi totalitari, e tra questi, il fascismo in particolare fece della difesa e salvaguardia della lingua ${ }^{31}$ nazionale una delle politiche di regime tendenti a identificare l'esaltazione patriottica e nazionalista con la costruzione di un'identità nazionale che fosse coincidente con il fascismo stesso. Da questo punto di vista i due nazionalismi linguistici, quello estadonovista e quello fascista, entrarono in aperta collisione ancor prima dell'entrata in guerra del Brasile a fianco degli anglo americani. L'uso strumentale della questione della lingua, come strategia geopolitica del regime fascista nei confronti delle comunità italiane all'estero, appare evidente in questa dichiarazione che Mussolini fece già nel 1920:

In cinquant'anni di vita, l'Italia ha realizzato progressi meravigliosi. Prima di tutto c'è un dato di fatto: ed è la vitalità della nostra stirpe, della nostra razza. [...] L'Italia faceva 27.000.000 di abitanti nel 1870; ne ha 50.000.000 adesso: 40.000 .000 nella penisola, ed è il blocco più omogeneo che ci sia in Europa. [...] E accanto a questi 40.000.000 in Italia, ce ne sono 10.000 .000 che hanno straripato in tutti i continenti, oltre tutti gli oceani: 700.000 italiani sono a Nuova York; 400.000 nello Stato di San Paolo dove la lingua di stato dovrà divenire la lingua italiana. ${ }^{32}$

Convertire la lingua italiana in lingua di stato a São Paulo nel 1920 era più una dichiarazione propagandistica, una sparata demagogica, che non un'aspirazione realizzabile o ragionevole, ma ci segnala quanto precoce fosse l'attenzione del fascismo verso la questione della lingua, se addirittura due anni prima della Marcia su Roma, Mussolini cercava di infiammare la piazza della riconquistata Trieste con simili affermazioni.

29. Ver HALL Michael, Imigrantes na cidade de São Paulo, in PORTA Paula, (a cura di), História da Cidade de São Paulo, vol. III, Paz e Terra, São Paulo 2004, p. 124. Per la distribuzione sul territorio dello stato di São Paulo suddivisa per nazionalità e decenni, cfr., BASSANEZI Maria Silvia Beozzo, População, imigração e propriedade da terra: procedimentos de pesquisa, "XV Encontro Nacional de Estudos Populacionais», ABEP, Caxambu - MG, 18-22 settembre 2006.

30. LEITE Aureliano, Italianos em São Paulo, «O Estado de São Paulo», 20 aprile 1954, in CARELLI Mario, Carcamanos e comendadores. Os italianos de São Paulo: da realidade á ficção (1919-1930), Ática, Sāo Paulo 1985, p. 31.

31. Sulla politica del fascismo in campo linguistico si vedano gli atti del convegno organizzato dal Centro Ligure di Storia Sociale dal 22 al 24 marzo 1984 a Genova coordinato da Lorenzo Coveri, Parlare fascista. Lingua del fascismo, politica linguistica del fascismo, in «Movimento Operaio e Socialista», n. 1, anno VII, 1984.

32. Cfr., Scritti e Discorsi di Benito Mussolini, Vol. II, La Rivoluzione Fascista (23 marzo 1919 - 28 ottobre 1922), Hoepli, Milano 1934, pp. 9596, discorso fatto a Trieste il 20 settembre del 1920, per il cinquantesimo anniversario della presa di Roma. 


\section{La culietta del caffé e la guerra italo-turca}

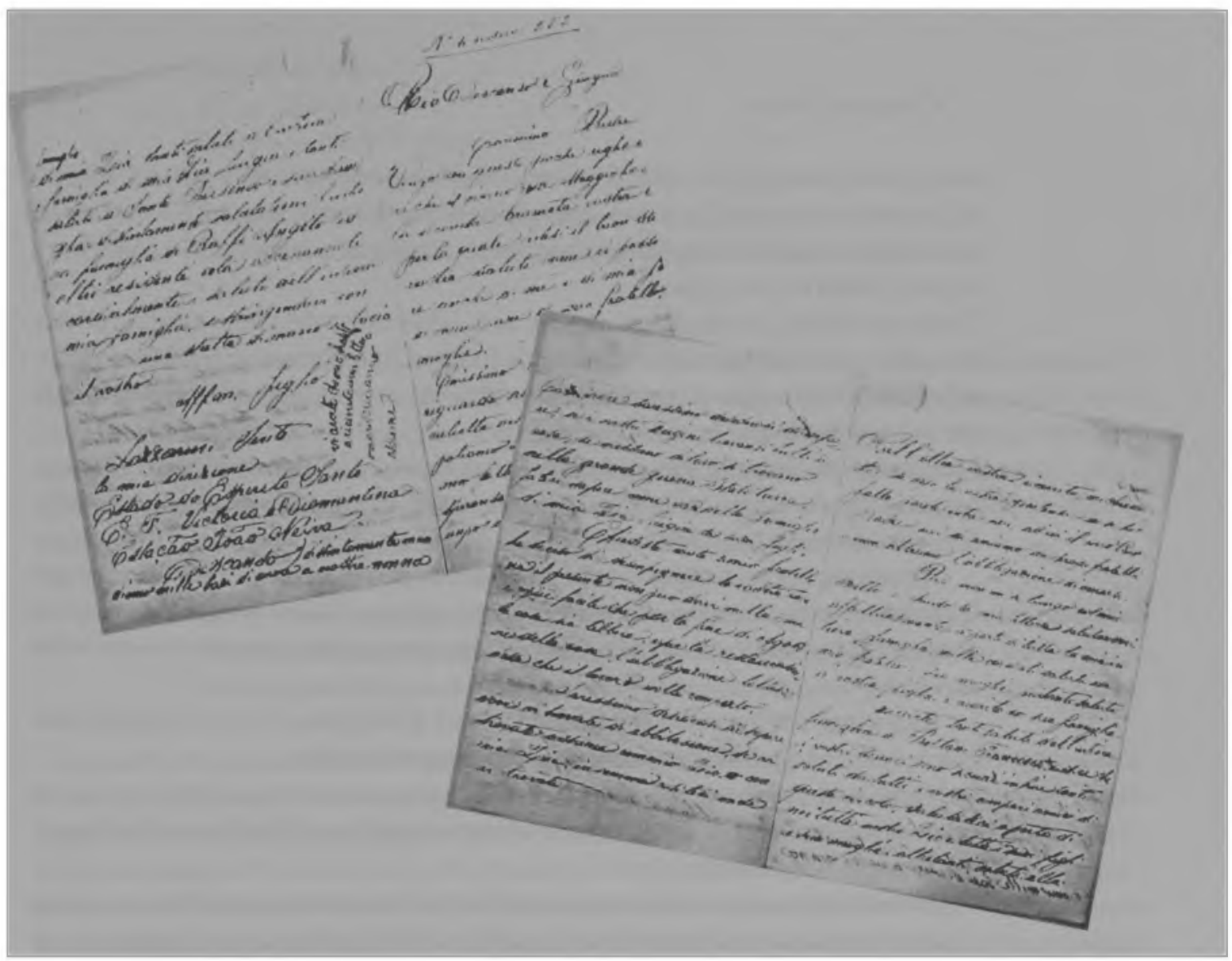

La lettera che presentiamo fa parte anch'essa del fondo delle Lettere di Chiamata del Memorial do Imigrante di São Paulo. Il destinatario della lettera è sbarcato nel porto di Santos con il Vapore Tommaso di Savoia il 4 luglio 1912. 


\section{Trascrizione}

Rio Descanso 2 giugno 1912

\section{Carissimo Padre}

Vengo con queste poche righe avvertendovi che il giorno rXde [illeggibile] Maggio ho ricevuto la seconda bramata vostra lettera per la quale intesi il buon stato di vostra salute come vi posso assicurare anche di me e di mia famiglia, e come pure di mio fratello e sua moglie.

Carissimo Padre, mi chiedete conto riguardo allarecolto del grano e della culietta del caffè. per l'arecolto non potiamo lamentarsi per i faciuoli ne abbiamo fatto abbastanza, ed il miglio abbiamo speransa di farne, per il caffe siamo unpò attrasadi, per causa di le pioggie [cambio pagina]

Caro Padre, saressimo desiderosi di sapere, se i nostri cugini trovansi tutti a casa, se nessuno di loro si trova nella grande guerra Italo-turca. fateci sapere come va della famiglia di mia zia Luigia dei suoi figli.

Chiedeste conto se mio fratello ha deciso di disimpegnare la vostra casa per il presente non può dirvi nulla, ma è più facile che per la fine di Agosto la casa sia libera, e per la restaurazione della casa l'ubbligazione toltasi desse che il lavoro volle compirlo.

Saressimo desiderosi di sapere dove vi trovate di abbitasione, se vi trovate assieme commio Zio o com mia Zia, insomma diteci onde vi trovate Qull'altra vostra ricevuta, mi chiedeste, se dopo la vostra partenza se ho fatto parole entre noi, adirvi il vero Caro padre noi ci amiamo come propi fratelli e come abbiamo l'obbligazione di amarsi,

Più non m’à lungo col mio scritto e chiudo la mia lettera salutandovi affettuosamente a parte di tutta la mia intiera famiglia, mille cordiali saluti da mio fratello e sua moglie, piùtanti saluti a vostra figlia e marito cò sua famiglia.

ricevete tanti saluti dall'intiera famiglia di Bottani Francesco, e dice che i vostri denari sono sicuri infine tanti saluti da tutti i vostri compari amici di questo circolo, salutateci a parte di tutti noi nostro Zio e i tutti i suoi figli e sua moglie, altretanti saluti alla [cambio pagina] famiglia di mia Zia tanti saluti a l'intiera famiglia di mia Zia Luigia e tanti saluti a Santo Parsendo e sua famiglia distintamente salutatemi l'intiera famiglia di Baffi Angelo ed altri residenti colà accenandoli cordialmente 
i saluti dell'intiera mia famiglia e stringendovi con una stretta di mano vi bacia il vostro Affm Figlio

[scritto di traverso] vi avverto che mio fratello à ricevuto una lettera maviscrivamo assieme

Lazzaroni Sante

la mia direzione

Estado do Espirito Santo

E.F. Victoria Diamantina

Estaçao Joao Neiva Descanso

distintamente mandiamo mille baci di cuore a nostra nonna

Appare immediatamente evidente che la struttura del testo è fortemente influenzata dalle formule tipiche della scrittura epistolare sia nei saluti di apertura ${ }^{33}$ (Vengo con queste poche righe) sia in quelli di chiusura (Più non mà lungo col mio scritto e chiudo la mia lettera salutandovi affettuosamente, distintamente mandiamo, distintamente salutatemi) dove sono presenti echi e rimandi di modelli scolastici acquisiti in modo incerto o improprio che generano un contrasto stridente dal punto di vista lessicale (la seconda bramata vostra lettera).

La rilevanza dei cliché e dell’approccio formulaico si spiega visibilmente con il peso che le espressioni fisse hanno nelle culture orali o semi-orali rispetto alle funzioni a loro attribuite invece nelle culture basate sulla scrittura o sulla stampa. ${ }^{34}$

L'incerta divisione delle parole è un altro degli elementi tipici della scrittura popolare (allarecolto, l'arecolto, unpò, maviscrivamo). Le interferenze lessicali del portoghese sono molte (attrasadi), ma in particolare appaiono significative quelle relative al lavoro dei campi, come se testimoniassero la sedimentazione di elementi di cultura locale che in lingua madre non avevano un corrispettivo culturalmente abbastanza forte e stabile da resistere a tali interferenze. La valenza sociolinguistica di espressioni come culietta del caffê, operazione che sicuramente non faceva parte delle abitudini contadine prima dell'arrivo in Brasile, o miglio per granoturco, hail potere di evocare trasformazioni culturali profonde in atto negli scriventi.

Anche la presenza di modelli burocratici imitati in modo goffo (trovansi, l'ubbligazione toltasi, residenti colà) è un altro elemento ricorrente nella scrittura popolare, tra quelli che abbiamo segnalato all'inizio di questo lavoro. ${ }^{35}$

33. Riguardo alla persistenza delle formule nella scrittura epistolare dei ceti subalterni hanno insistito tutti gli studi, a partire dai classici di THOMAS William Isaac e ZNANIECKI Florian, Il contadino polacco in Europa e in America, cit; e SPITZER Leo, Lettere di prigionieri di guerra italiani, cit. Le conclusioni a cui gli autori sono pervenuti, percorrendo strade diverse ed analizzando generi e contesti geografici e culturali fra loro molto distanti, presentano molte analogie e confermano ulteriormente la tesi dell'uniformità di fondo delle corrispondenze popolari. Ma come spesso accade, pur rimanendo dei punti di riferimento fondamentali per chiunque si voglia avvicinare allo studio di questi documenti, questi celebri studi hanno anche, in qualche misura, contribuito alla formazione di pregiudizi e stereotipi - l'uniformità e la formularietà è tale da rendere questi documenti praticamente tutti uguali e inutili per il ricercatore, sia esso storico o linguista - che ancora oggi pesano su questo genere di documenti.

34. FRANZINA Emilio, L'epistolografia popolare e i suoi usi, in Per un Archivio della scrittura popolare, «Materiali di lavoro», n. 1-2, 1987, pp. 27-28.

35. In particolare cfr. CORTELLAZZO Manlio, Avviamento critico allo studio della dialettologia italiana, cit.; e BERRUTO Gaetano, L'Italiano popolare, cit. 
L'importanza di ricevere posta e mantenere saldi i legami familiari attraverso la corrispondenza è ben messa in evidenza dall'espressione Saressimo desiderosi di sapere -reinvenzione creativa e straordinariamente suggestiva di un condizionale che riesce a comunicarci direttamente l'ansia di notizie che poteva attraversare gli oceani, ripetuta per due volte, nonché dallo spazio dedicato all'elencodelle persone da salutare che occupa quasiuna pagina e mezza dell'intero testo. L'interferenza grafica del portoghese è evidente in espressioni come commio Zio o com mia Zia. Che parte del testo sia stata aggiunta dopo, e scritta di traverso è un altro elemento tipico di questi documenti, in cui gli aspetti grafici e materiali hanno una rilevanza notevole nell'aiutarci ad identificare il grado di dimestichezza con il mezzo scrittorio dellautore. La sostituzione della $\mathrm{z}$ con la $\mathrm{s}$ in speransa $\mathrm{e}$ abbitasione, è altra interferenza del portoghese o forse il retaggio di un sostrato dialettale tipico del nord-est padano (ma non abbiamo dati certi sulla provenienza regionale dello scrivente), già notato nel caso dei soldati della Grande guerra. Un'ultima questione di particolare importanza resta da segnalare: la preoccupazione per sapere se qualche parente è partito per la grande guerra Italo-turca. Par di intravvedere l'eco della propaganda di guerra che la stampa in lingua italiana riportava a São Paulo: l'evento non viene citato come guerra di Libia, ma appunto come grande guerra Italo-turca, ossia la dizione che veniva riportata sulle prime pagine della stampa locale, la prima grande mobilitazione nazionale che ha visto schierati a favore della guerra intellettuali e poeti internazionalmente noti e soprattutto fino ad allora schierati con il movimento socialista, com’è stato il caso di Giovanni Pascoli.Ciò significa che l'attenzione allequestioni politiche internazionali e italiane in particolare era costante, cosi come il livello di informazione attualizzato sia attraverso la stampa, sia attraverso la corrispondenza.

L'analisi di questa lettera è solo un esempio della ricchezza e delle possibilità offerte da una fonte di questo tipo. Avere a disposizione un serbatoio di materiale così ricco è un'occasione straordinaria per cominciare a ragionare sui mutamenti delle lingue a contatto anche dal punto di vista della scrittura e non solo del parlato, in maniera non episodica. Il fondo delle Lettere di Chiamata, offrendoci la possibilità di analizzare una quantità considerevole di documenti, indubbiamente dei frammenti, delle schegge di quell'enorme flusso di carta che è stato scambiato fra l'Italia e il Brasile - ma la serialità in questo genere di documenti è impossibile e, in certa misura, inutile, proprio perché lo studio che se ne può fare è sempre di carattere qualitativo - può diventare l'occasione per addentrarsi in profondità nei meccanismi di contaminazione e cambiamento linguistico di cui i processi migratori sono un potente acceleratore. 


\section{Bibliografia}

BANFI Emanuele, Analisi variazionistiche nelle lettere di un migrante ladino in Brasile a metà '800, in Id. e CORDIN Patrizia (a cura di), Pagine di scuola, di famiglia, di memorie. Per una analisi del multilinguismo nel Trentino austriaco, Museo Storico in Trento, Trento, 1996.

BARTOLI LANGELI Attilio, La scrittura dell'italiano, Il Mulino Bologna 2000.

BASSANEZI Maria Silvia Beozzo, População, imigração e propriedade da terra: procedimentos de pesquisa, «XV Encontro Nacional de Estudos Populacionais», ABEP, Caxambu - MG, 18-22 settembre 2006.

BENDOCCHI Alves Débora, Cartas de imigrantes como fonte para o historiador: Rio de Janeiro-Turingia (1852-1853), in «Revista Brasileira de História», n. 45, 2003, pp. 155-184.

BERRUTO Gaetano, L'Italiano popolare, in IDEM, Lineamenti di sociolinguistica dell'italiano contemporaneo, La nuova Italia Scientifica, Roma 1987, pp. 107-140.

BRUNI Francesco (a cura di), L'italiano nelle regioni. Lingua nazionale e identità regionali, Utet, Torino 1992.

BRUNI Francesco (a cura di), L'italiano nelle regioni. Lingua nazionale e identità regionali, Utet, Torino 1992.

BUENO Alexandre Marcelo, Intolerância Lingüística e Imigração, 2006, Tese de Mestrado do Programa de Pósgraduação em Semiótica e Lingüística Geral da FFLCH-USP, orientadora Profa Dra, Diana Luz Pessoa De Barro.

CARDONA Giorgio Raimondo, Introduzione all'etnolinguistica, Il Mulino, Bologna 1976.

CARELli Mario, Carcamanos e comendadores. Os italianos de Sáo Paulo: da realidade à ficção (1919-1930), Ática, São Paulo 1985.

CHEDA Giorgio, L'emigrazione Ticinese in Australia, Armando Dadò Editore, Locarno 1979.

CHEDA Giorgio, L'emigrazione Ticinese in California, Armando Dadò Editore, Locarno, 1981.

CORTELlaZZO Manlio, Avviamento critico allo studio della dialettologia italiana, Vol. III, Lineamenti di italiano popolare, Pacini, Pisa 1972.

COVERI Lorenzo, Italiano popolare, scrittura popolare: una prospettiva linguistica, in «Materiali di lavoro», n. 1-2, 1987.

CROCI Federico e BONFIGLIO Giovanni, El baúl de la memoria. Testimonios escritos de inmigrantes italianos en el Perú, Fondo Editorial del Congreso de la República del Perú, Lima 2002.

CROCI Federico, Modernizzazione e pratiche comunicative. La scrittura dell'italiano nel XX secolo, in «Italianistica», n. XII, in particolare pp. 115-132.

CROCI Federico, Scrivere per non morire. Lettere dalla Grande Guerra del soldato bresciano Francesco Ferrari, Marietti (ora Paravia-Scriptorium) Genova 1992.

DAVATZ Thomas, Memórias de um colono no Brasil: 1850, Edusp, São Paulo 1980 (con prefazione e note di Sergio BUARQUE DE HOLLANDA).

DE AMICIS Edmondo, Sull'Oceano, (a cura di BERTONE Giorgio), Diabasis, Reggio Emilia, 2005.

DE MAURO Tullio, Storia linguistica dell'Italia unita, Laterza, Roma-Bari $2005^{9}$.

DEVOTO Fernando, Algo más sobre las cadenas migratorias de los italianos a la Argentina, in «Estudios Migratorios Latinoamericanos», n.19, 1991.

DEVOTO Fernando, Le migrazioni italiane in Argentina. Un saggio interpretativo, Officina Tipografica, Napoli 1994

DEVOTO Fernando, Liguri nell'America australe: reti sociali, immagini, identità, in RUGAFIORI Paride e GIBELLI Antonio (a cura di), Storia d'Italia, Le regioni, dall'unità a oggi. La Liguria, Einaudi, Torino 1994, pp. $651-688$.

FABIETTI Ugo, Storia dell'antropologia, Zanichelli, Bologna 2001.

FAUSTO Boris e DEVOTO Fernando, Brasil e Argentina: um ensaio de história comparada (1850-2002), Editora 34, São Paulo $2005^{2}$

FRANZINA Emilio, L'epistolografia popolare e i suoi usi, in Per un Archivio della scrittura popolare, "Materiali di lavoro», n. 1-2, 1987, pp. 21-76.

FRANZINA Emilio, L'immaginario degli emigranti. Miti e raffigurazioni dell'esperienza italiana all'estero fra i due secoli, Pagus, Treviso 1992. 
FRANZINA Emilio, Merica! Merica Emigrazione e colonizzazione nelle lettere dei contadini veneti e friulani in America Latina (1876-1902), Milano, Feltrinelli, 1979 (Verona, Cierre Edizioni, 1994²).

GIBELLI Antonio e CAFFARENA Fabio, Le lettere degli emigranti, in BEVILACQUA Piero a, DE CLEMENTI Andreina e FRANZINA Emilio (a cura di), Storia dell'emigrazione italiana, Vol. I, Partenze, Roma, Donzelli, 2001, pp. 563-574.

GIBELLI Antonio, Il popolo bambino. Infanzia e nazione dalla Grande Guerra a Salò, Einaudi, Torino 2005, pp. 277-290.

GIBELLI Antonio, L'officina della guerra, La Grande Guerra e le trasformazioni del mondo mentale, Bollati Boringhieri, Torino $1998^{2}$

HALL Michael, Imigrantes na cidade de São Paulo, in PORTA Paula, (a cura di), História da Cidade de São Paulo, vol. III, Paz e Terra, São Paulo 2004.

HORN IOTTI Luiza (a cura di), Imigração e colonização, legislação 1747 a 1915, Educs, Caxias do Sul 2001.

LONGO di Cristofaro Gioia, MARIOTTI Luciana (a cura di), Modelli culturali e differenza di genere, Armando, Roma 1998.

LUSSANA Filippo, Lettere di illetterati. Note di psicologia sociale, Zanichelii, Bologna s.d. [1913].

MACHADO CAMPOS Cynthia, A política da lingua na era Vargas. Proibição do falar alemäo e resistências no Sul do Brasil, UNICAMP, Campinas 2006.

MALINOWSKY Bronislav, Teoria scientifica della cultura, Feltrinelli, Milano 1962.

MEZZADRA Sandro, Diritto di fuga. Migrazioni, cittadinanza e globalizzazione, Ombre Corte, Verona 2006.

Parlare fascista. Lingua del fascismo, politica linguistica del fascismo, inserto monografico in «Movimento Operaio e Socialista", n. 1, anno VII, 1984.

PETRUCCI Armando, Scrivere lettere. Una storia plurimillenaria, Laterza, Roma-Bari 2008.

RAMELLA Franco, Reti sociali, famiglie e strategie migratorie, in BEVILACQUA Piero, DE CLEMENTI Andreina, FRANZINA Emilio (a cura di), Storia dell'emigrazione italiana, Vol. 1, Partenze, cit., pp. 143-160.

SERVETTI Lorenza, Vado nella Merica: è lì di là dalle colline. Budrio e la grande emigrazione (1880-1912), Marsilio, Venezia 2003.

SERVETTI Lorenza, Vado nella Merica: è lì di là dalle colline. Budrio e la grande emigrazione (1880-1912), Marsilio, Venezia 2003.

SPITZER Leo, Lettere di prigionieri diguerra italiani 1915-1918, Boringhieri, Torino 1976.

THOMAS William Isaac e ZNANIECKI Florian, Il contadino polacco in Europa e in America, Edizioni Comunità, Milano 1968, 2 voll..

VANGELISTA Chiara, Os Braços da lavoura. Imigrantes e caipiras na formação do mercado de trabalho paulista (1850-1930), São Paulo, HUCITEC 1991.

VEDOVELLI Massimo, L'italiano degli stranieri, Carocci, Roma 2002.

ZADRA Camilio e FAIT Gianluigi (a cura di), Deferenza. Rivendicazione. Supplica. Lettere ai potenti, Treviso, Pagus Edizioni, 1991.

\section{Fonti}

Arquivo do Estado de São Paulo [AESP], serie Inspetoria de Terras e Colonização, Boletim da Diretoria de Terras, Colonização, e Imigração.

Arquivo do Estado de São Paulo [AESP], serie Secretaria da Agricultura, Departamento de Imigração e Colonização, Estatística dos trabalhos executados pelo Departamento de Imigração e Colonização durante o ano de 1961, São Paulo, 1962.

Arquivo do Estado de São Paulo [AESP], serie Secretaria da Agricultura 1823-1926, Comércio e Obras Públicas, Comunicado de 13 de fevereiro de 1909.

Arquivo do Memorial do Imigrante [AMI], São Paulo, Fondo Documentos Pessoais, Cartas de Chamada.

MUSSOLINI Benito, Scritti e Discorsi di Benito Mussolini, Vol. II, La Rivoluzione Fascista (23 marzo 1919 - 28 ottobre 1922), Hoepli, Milano 1934. 